\title{
GENDER DIFFERENCES IN PREDICTING UNSAFE DRIVING BEHAVIORS IN YOUNG ADULTS
}

\author{
Nicole R. Skaar, John E. Williams \\ University of Northern Iowa \\ Cedar Falls, Iowa, USA \\ E-mail: nskaar24@uni.edu
}

\begin{abstract}
Summary: Past research has uncovered personality influences on dangerous driving behavior and vehicle crashes (Furnham \& Saipe, 1993; Matthews, Dorn, \& Glendon, 1991). Recently, females between 16 and 20 years of age showed an increase in overall crash rate, while males within the same age group showed a decrease in overall crash rate (NHTSA, 2004; NHTSA, 2002). Adolescent and young adult females have become a critical cohort in the study of unsafe driving behavior. The purpose of this study was to examine gender differences in risky driving behavior and personality influences on these behaviors. Data presented are first year cross-sectional results in a 5-year longitudinal study. Participants were 141 male and female drivers who completed the NEO PI-R, the DDDI and a driving behavior questionnaire. In addition, the driving record of each participant was obtained from the State Department of Transportation. Females reported a higher rate of traffic violations and crashes than males. No personality traits were significantly related to crashes, but Extroversion was positively related to total traffic violations within females. The DDDI scales were not significantly related to traffic violations or vehicle crashes, but gender differences were shown within the risky driving and aggressive driving scales. Gender differences were also shown in the relationship between personality traits and DDDI scales. This data indicates that researchers and insurance companies should make an effort to consider females in their work. In addition, this study provides preliminary data on gender differences in personality as it relates to risky driving behavior.
\end{abstract}

\section{OBJECTIVES}

Over 60 years ago, research found that automobile crashes were strongly linked to driver behavior such as speeding, cutting in, and refusing to yield the right of way (Ross, 1940). More recent research has uncovered personality influences on dangerous driving behavior. Vavrik (1997) studied male drivers between the ages of 16 and 21 and found that a group of drivers with two or more vehicle crashes differed significantly on measures of affiliation, desirability, dominance, exhibition, and self-esteem than a group of drivers with no at-fault vehicle crashes. Furnham and Saipe (1993) studied personality correlates of risky driving and found that drivers who had multiple driving infractions scored higher on a scale of Psychoticism and lower on a scale measuring Neuroticism. None of the personality measures in this study were significantly correlated with crashes, but other research has found Neuroticism to be related to vehicle accidents (Matthews, Dorn, \& Glendon, 1991). In addition, Arthur \& Graziano (1990) found conscientiousness to be inversely related to vehicle crash involvement and number of moving violations in a sample of college students. 
It is important to study driving correlates within adolescents and young adults because drivers between the ages of 16 and 20 have the highest fatality and injury rates in the United States (NHTSA, 2004). Even though the statistics continue to show males at higher risk for fatal and injurious crashes, females between 16 and 20 years of age had an increase in overall crash rate from 2000 to 2002, while males within the same cohort had a decrease in overall crash rate (NHTSA, 2004; NHTSA, 2002). This trend continued in 2003, with a $9 \%$ increase in driver fatalities for young males, while female drivers had a 25\% increase in fatal crashes (NHTSA, 2005). Adolescent and young adult females have become a critical cohort in the study of unsafe driving behavior.

Boyce and Geller (2002) looked at personality in both male and female drivers of all ages and found a significant relationship between driver age and unsafe driving behaviors such as speeding and short following distance (the youngest and the oldest drivers engaging in more unsafe behaviors than the middle ages), but found no gender differences. The female drivers had just as many risky driving behaviors, such as speeding and close following, as the male drivers in the study. They also found Type A personality to be a significant predictor of risky driving as these participants drove faster than those subjects not described as Type A. This is not a surprise as Type A people are characterized as impatient and competitive (Eysenck \& Fulker, 1984).

The literature also shows positive relationships between other personality measures and risky driving. Sensation seeking is found to be related to number of speeding tickets, fast driving, rule violations, and self assertiveness (Ullberg, 2001; Whissell \& Bigelow, 2003; Arnett, 1996). Arnett (1996) also found aggressiveness to have a significant positive correlation with risky driving behaviors such as speeding, driving while intoxicated, and passing in a no-passing zone. There is also some evidence that increased impulsivity plays a role in risky driving behavior (Wilson, 1990; Stanford et al., 1996).

There are many measures of normal personality within the literature; some measure a broad range of personality traits while others measure more specific traits. Zuckerman's (1979) sensation seeking scale, Type A personality measures, and measures of impulsivity are tests designed to look at a specific trait. Other measures such as the 16PF (Cattell, 1993), the Eysenck Personality Questionnaire (1975), and the NEO PI-R (Costa \& McCrae, 1992) measure both global personality traits along with specific traits, which leads to a more comprehensive personality measure. The present study uses a global measure of personality, the NEO PI-R, which provides a global personality assessment as well as measures of specific personality traits. This allows us to look at several possible correlates of risky driving; for example, the Extroversion scale will measure overall Extroversion along with excitement-seeking, positive emotions, activity and assertiveness. Within other facet scales we will measure hostility, impulsiveness, compliance, self-discipline, and dutifulness.

A recent addition to the available measures of risky driving is the Dula Dangerous Driving Index (DDDI). The DDDI was developed specifically to assess for dangerous driving risk. This instrument combines self reported behaviors and emotions related to driving. The developers found that the total dangerous driving scale was positively related to traffic citations and at-fault crashes. They also found gender differences in aggressive and risky driving with males reporting higher levels of this type of driving than females; but females and males reported comparable amounts of dangerous driving and negative emotions while driving (Dula \& Ballard, 2003). We 
aimed to replicate Dula's previous work and to expand it by looking at the relationship between the DDDI and personality traits.

There is an abundance of research showing that various personality traits are linked to risky driving behavior and vehicle crashes. This study expands past recent work by investigating gender differences within personality predictors. The purpose of this study was to examine gender differences in driving behavior, traffic violations, propensity for dangerous driving, and personality traits that predict these driving behaviors.

\section{METHODS}

\section{Participants}

Participants were 141 drivers (30.5\% male, 69.5\% female) between the ages of 16 and 22 $(\mathrm{M}=17.96, \mathrm{SD}=1.34)$. Education range was between 10 and 15 years $(\mathrm{M}=11.71, \mathrm{SD}=1.15)$. Participants were recruited from the student population at a small Midwestern university and from two high schools in Minnesota and Iowa. The high schools were chosen based on availability of the students and size of the school. We recruited students from a small rural community and from a larger, more metropolitan community in order to sample a wide range of driver training and experience.

\section{Procedures}

Consent was obtained from each participant and from the participant's parent or guardian when necessary. Each participant was administered an array of self-report personality and behavioral questionnaires. We also obtained the driving record of each Iowa participant from the Iowa Department of Transportation in order to cross reference traffic violations and crashes with the self-report. A detailed account of all crashes was obtained from each participant and read by two researchers in order to determine fault. This data was gathered from the participant because access to crash reports in the state of Iowa is restricted to the attorney general, the driver's insurance company, and those working within the state DOT. This is a 5-year longitudinal study where participants will be administered the same self-report questionnaires each year. An updated driving record will also be obtained each year from both the Iowa and Minnesota Departments of Transportation.

\section{Measures}

NEO Revised Personality Inventory (NEO PI-R). The NEO PI-R is a self-report inventory based on the Five Factor Model of personality. Responses are made on a 5-point Likert scale ranging from strongly disagree to strongly agree. Internal reliabilities for the five factors (Agreeableness, Conscientiousness, Openness, Extraversion and Neuroticism) are 0.76, 0.86, 0.89, 0.87, and 0.93 , respectively. Validity of the NEO-PI-R was examined by comparing the five domains with other adjectives $(\mathrm{r}=0.56-0.62)$, spouse ratings $(\mathrm{r}=0.44-0.65)$, and peer ratings $(\mathrm{r}=0.33-$ 0.48) (Costa \& McCrae, 1992).

Dula Dangerous Driving Index (DDDI). The DDDI was developed to measure the likelihood of driving dangerously based on the driver's self report of driving behaviors and emotion while 
driving. Each of the subscales (Dangerous Driving Total, Aggressive Driving, Negative Emotional Driving, and Risky Driving) was found to have excellent internal reliability ( 0.83 to 0.92). Temporal reliability is adequate, ranging from 0.55 on the Aggressive Driving Scale to 0.76 on the Risky Driving Scale (Dula \& Ballard, 2003).

Driving Behavior Questionnaire. The Driving Behavior Questionnaire was developed for the purpose of this study. It included questions regarding speeding tickets, other traffic violations, crashes in which the participant was the driver, and license suspension/revocation. Participants were asked to list the dates of the above driving violations within their lifetime, and also were asked to describe, in detail, vehicle crashes and reasons for license suspension/revocation.

\section{Data Analysis}

The data used in the analysis are the first year cross-sectional data from a larger project. Total number of traffic violations was calculated as a sum of total speeding tickets, total other traffic violations, total crashes, and total license suspensions/revocations. The NEO PI-R gives several measures, including total scores and six facet scores for each of the five scales (Neuroticism, Extroversion, Openness, Agreeableness, and Conscientiousness). Data analysis included comparison of means t tests, Pearson correlational analysis and stepwise regression analysis.

\section{RESULTS}

\section{Gender Differences}

The results showed that even though the number of female drivers involved in at least one atfault vehicle crash did not differ significantly from males $(t=-1.63, p=0.105)$, females did have a significantly greater number of at-fault crashes than did the male drivers $(t=2.329, p=0.022)$. Females also showed a significantly greater number of traffic violations than males $(\mathrm{t}=2.291, \mathrm{p}$ $=0.024)$. Females had a total traffic violation rate of $54.1 \%$, while males had a total traffic violation rate of $42.8 \%$. Females showed a total crash rate of $33.3 \%$, while males showed a total crash rate of only $19.5 \%$.

Of the five personality traits measured, only Extroversion showed a significant correlation with total number of traffic violations $(r=0.233, p=0.022)$ and this was only significant for females. Stepwise linear regression also revealed extroversion to be the only predictor of total traffic violations, but only for females $(\beta=0.231, p=0.023)$. Within the 6 facet scores of extroversion, activity was the only predictor of total traffic violations $(\beta=0.301, p=.003)$, and this was also shown only in females. No personality trait showed a significant relationship with number of crashes, but extroversion did approach significance within females $(r=0.176, p=0.087)$.

\section{DDDI Validation Data}

None of the DDDI scales showed a significant correlation with either total traffic violations or at-fault crashes. As expected, no gender differences were found for the DDDI total or DDDI negative emotions, but males did have significantly higher scores on the aggressive driving scale $(\mathrm{t}=-2.286, \mathrm{p}=0.024)$ and the risky driving scale $(\mathrm{t}=-2.962, \mathrm{p}=0.004)$. Gender differences were also found in the relationship between personality traits and the DDDI scales in that high 
Neuroticism $(r=0.272, p=0.007)$, low Agreeableness $(r=-0.321, p=0.001)$, and low Conscientiousness $(\mathrm{r}=-0.364, \mathrm{p}=<0.001)$ were significantly correlated across the DDDI scales in females. The DDDI total score was not significantly correlated to any of the personality scales, but Neuroticism showed a significant positive correlation with negative emotions $(\mathrm{r}=$ 0.357, $\mathrm{p}=0.024)$, Openness, Agreeableness and Conscientiousness showed significant negative correlations with negative emotions $(\mathrm{r}=-0.387, \mathrm{p}=0.013)$, aggressive driving $(\mathrm{r}=-0.325, \mathrm{p}=$ $0.041)$ and risky driving $(\mathrm{r}=-0.385, \mathrm{p}=0.014)$ scales respectively.

\section{CONCLUSIONS}

As expected, the results indicate that young female drivers have a high incidence of at-fault vehicle crashes and traffic violations, but unlike the national statistics, females in the present study exceeded the males in both traffic violation rate and crash rate. Researchers and insurance companies need to make an effort to consider females in their work and put aside the common stereotype that males are more prone to vehicle crashes than females. Insurance companies use actuarial data to determine insurance premiums, and at the present time young males have higher insurance premiums than young females. There is some discussion within insurance companies that there should be increased parity among young male and female insurance premiums in the coming years (Drahn, 2005).

Extroversion was a significant predictor for traffic violation within females, but it was activity, not excitement seeking, that fueled the prediction ability of the scale. Females who are characterized as fast paced, high energy and busy are those who will incur more traffic violations, and possibly crashes, than those who are characterized as more relaxed. It is uncertain whether this significant finding is a true gender difference in personality predictors of risky driving or if the finding is due to the low number of males in the sample.

Ulleberg (2001) studied both personality and attitudes of risky drivers and found that personality traits influenced driving indirectly by affecting attitudes of the drivers. This indirect relationship between personality and driving behavior is a potential explanation for the results of this study. Miles and Johnson (2003) found no significant differences between a known sample of aggressive drivers and a student sample in personality traits of agreeableness, conscientiousness, and neuroticism. The body of research is currently inconclusive and segmented. Future directions of research in personality and driving might focus on the use of instrumented vehicles and driving simulators in addition to well-validated personality instruments and self-reported driving behavior. A meta analytic examination of the current published research may also illuminate the relationship between personality and risky driving.

The results also show clear gender differences in the prediction of self-reported propensity for dangerous driving. While higher tendency to experience negative affect correlates with female unsafe driving behavior, low intellectual curiosity correlates with male risk for dangerous driving behavior. Even though this study did not show the significant relationship between DDDI scales and traffic citations found in other studies (Dula \& Ballard, 2003), this important research needs to be replicated to ensure validity of the instrument.

Personality inventories and the DDDI could potentially be used in driver education programs to predict unsafe drivers before they get on the road. We know that personality is fairly stable after 
the age of 30 (McCrae \& Costa, 1994), but there may be room for modification during adolescence. Driver education students with personality indicators of future unsafe driving behavior could be targeted for interventions. There are three cognitive-behavioral interventions that have been indicated for aggressive driver: one-day intensive cognitive-behavioral therapy, group relaxation/cognitive therapy, and brief cognitive-behavioral therapy that focus on relaxation skills, coping skills and changing aggressive belief systems. These interventions have shown positive outcomes in both self-referred volunteers and court-mandated participants (Galovski \& Blanchard, 2004).

There are limitations to this study. The sample contained fewer males than females, and it is part of the ongoing nature of this study to gather more data with male participants. While some research calls attention to impression management (Lajunen et al, 1997), others have shown that self-report measures are quite accurate (Elander, West \& French, 1993). State archival data can also be inaccurate. The Iowa Department of Transportation records only crashes that lead to $\$ 1,000$ or more of damager to a vehicle. Moreover, there are crashes that are never reported to the state because (1) the police may have never been called to the scene and/or (2) the driver(s) involved in the crash may never have reported the crash to the state as requested. Another limitation is that this is a cross-sectional design and cannot confidentially predict traffic violations and vehicle crashes. Future longitudinal data will better elucidate the role personality plays in traffic violations and vehicle crashes (Caspi, Harrington, Moffitt, Begg, Dickson, Langley, et al., 1997).

\section{REFERENCES}

Arthur, W. \& Graziano, W. G. (1996). The Five Factor Model, conscientiousness, and driving accident involvement. Journal of Personality, 64(3): 593-618.

Boyce, T.E. \& Geller, S. (2002) An instrumented vehicle assessment of problem behavior and driving style: Do younger males really take more risks? Accident Analysis \& Prevention, 34, (1): 51-64.

Caspi, A., Harrington, H., Moffitt, T., Begg, D., Dickson, N., Langley, J., et al. (1997).

Personality differences predict health-risk behaviors in young adulthood: Evidence from a longitudinal study. Journal of Personality and Social Psychology, 73(5): 1052-1063.

Cattell, R.B., Cattell, A.K., \& Cattell, H.E. (1993). Sixteen personality factor questionnaire (5th ed.). Champaign, IL: Institute for Personality and Ability Testing.

Costa, P.T. Jr. \& McCrae, R.R. (1992). Revised NEO Personality Inventory (NEO-PI-R) and NEO Five Factor Inventory professional manual. Odessa, FL: Psychological Assessment Resources, Inc.

Drahn, J. Personal communication, April 12, 2005.

Dula, C.S. \& Ballard, M.E. (2003). Development and evaluation of a measure of dangerous, aggressive, negative emotional and risky driving. Journal of Applied Social Psychology, 33(2): 263-282.

Elander, J., West, R., \& French, D. (1993). Behavioral correlates of individual differences in road-traffic crash risk: An examination of methods and findings. Psychological Bulletin, 113: 279-294. 
Eysenck, H. J. \& Eysenck, S. B. (1975). Manual of the Eysenck Personality Questionnaire. London: Hodder and soughton.

Eysenck, H. J. \& Fulker, D. W. (1984). The components of Type A behavior and its genetic determinants. Personality and Individual Differences, 4(5): 499-505.

Galovski, T.E. \& Blanchard, E.B. (2004). Road rage: A domain for psychological intervention? Aggression and Violent Behavior, 9: 105-127.

McCrae, R.R. \& Costa, P.T. Jr. (1994). The stability of personality: Observations and evaluations. Current Directions in Psychological Science, 3: 172-175.

National Highway Traffic Safety Administration. (2005). Traffic Safety Facts 2003. Retrieved March 13, 2005, from NHTSA Web site: http://www-nrd.nhtsa.dot.gov/pdf/nrd30/NCSA/TSFAnn/TSF2003Final.pdf

National Highway Traffic Safety Administration. (2004). Traffic Safety Facts 2002. Retrieved March 1, 2004, from NHTSA Web site: http://www-nrd.nhtsa.dot.gov/pdf/nrd30/NCSA/ TSFAnn/TSF2002Final.pdf

National Highway Traffic Safety Administration (2002) Traffic Safety Facts 2000 Retrieved March 1, 2004, from NHTSA Web site: http://www-nrd.nhtsa.dot.gov/pdf/nrd30/NCSA/ TSFAnn/TSF2000.pdf

Ross, H. L. (1940). Traffic accidents: a product of social-psychological conditions. Social Forces, 18: 569-576.

Ulleberg, P. (2001). Personality subtypes of young drivers. Relationship to risk-taking preferences, accident involvement, and response to a traffic safety campaign. Transportation Research Part F: Traffic Psychology and Behaviour, 4(4): 279-297.

Vavrik, John. (1997). Personality and risk-taking: a brief report on adolescent male drivers. Journal of Adolescence, 20: 461-465.

Whissel, R. W. \& Bigelow, B. J. (2003). The Speeding Attitude Scale and the role of sensation seeking in profiling young drivers at risk. Risk Analysis, 23(4): 811-820.

Zuckerman, M. (1979). Sensation seeking: beyond the optimal level of arousal. Hillsdale, NJ: Erlbaum. 